\title{
Calculation of a Lower Bound Ratchet Limit Part 1 - Theory, Numerical Implementation and Verification
}

\author{
Haofeng Chen ${ }^{a}$, James Ure ${ }^{a}$, David Tipping ${ }^{b}$
}

${ }^{a}$ Dept of Mechanical and Aerospace Engineering, University of Strathclyde, Glasgow, Scotland, G1 1XJ
${ }^{b}$ Central Engineering Support, EDF Energy Nuclear Generation Ltd., Barnwood, Gloucester, GL4 3RS

\begin{abstract}
It is important to be able to calculate the ratchet limit of a component when performing integrity assessments of plant components. This paper details the addition of a lower bound ratchet limit calculation to the Linear Matching Method. The extension of Melan's theorem into the alternating plasticity region is explained, followed by its implementation into the Linear Matching Method calculation procedure. Finally, the convergence properties of this method are analysed by the analysis of a plate with a central hole subject to cyclic thermal and mechanical loading
\end{abstract}

Keywords: Shakedown, Ratchet limit, Lower bound, Linear Matching Method

\section{Introduction}

The ability to accurately calculate the shakedown and ratchet limits of plant components subject to cyclic loading is of ever increasing importance in many industries. Increasingly accurate shakedown and ratchet limit solutions are of particular importance to life extension in the nuclear industry; where comprehensive justification is required to prove that components are safe to operate beyond their initial design life.

Shakedown, where the component exhibits entirely elastic behaviour after initial plastic straining, is a well established phenomenon and is included in all pressure vessel design codes. The need for more accurate shakedown assessment has meant that many numerical methods have been developed in recent years which can directly predict the shakedown limit. These methods avoid the trial and error nature of traditional cyclic finite element analysis by making use of the bounding theorems of Koiter (1960) and Melan (1936). Such methods include nonlinear superposition (Hamilton et al, 2002), the GLOSS r-node method (Seshadri, 1995), the Elastic Compensation method (Mackenzie et al, 2000) and mathematical programming methods (Liu et al, 1997; Bocciarelli et al, 2004). In recent years mathematical programming methods have been extensively developed by numerous research groups, for example the extension by Simon and Weichart (2011) to include multiple load extremes in the load cycle. Mathematical programming methods have also been used by the LISA project (Staat and Heitzer, 2003). The numerous European research groups involved with the LISA project studied extensions to traditional shakedown analysis including kinematic hardening, damaged materials and large deformations.

In prominent structural assessment procedures, such as the UK nuclear industry's R5 (Ainsworth, 2003), components are allowed to operate beyond their traditional shakedown limits as long as "global shakedown" is satisfied. This is where the plastic strains in some regions of the structure form either an alternating plasticity mechanism or an elastic shakedown mechanism. If further assessment can prove that the plastic straining caused by the alternating plasticity will not cause failure by low cycle fatigue, then it is safe for the component to operate in this state. 
The Linear Matching Method is relatively unique among the direct methods mentioned as it has the capability to assess the ratchet limit, and so can determine whether a component is within global shakedown or not. In addition to including the effects of temperature dependent material properties, this method has recently been extended to include any number of extremes in the load cycle (Chen and Ponter, 2010). The LMM ratchet assessment procedure also calculates the plastic strain range, which can then be used to perform a low cycle fatigue assessment.

In terms of the UK nuclear industry, conservatism is a key issue and lower bound shakedown and ratchet limits are preferable in any analysis undertaken. For a shakedown assessment this means using Melan's theorem to ensure all stresses satisfy yield and thus ensure conservatism in the solution. The drawback with lower bound methods, based on Melan's theorem, is that the high stress at discontinuities and concentrations can create convergence problems as the redistribution of these localised stresses defines the final limit. These convergence issues arise because the solution depends on every integration point in the finite element model satisfying the yield condition at all points in the load cycle. The finite element method calculates displacements fields, and then the stresses which arise due to this are a secondary calculation, leading to slight inaccuracies which can affect lower bounds. If even a single integration point does not satisfy yield then the lower bound solution will be dictated by this point. Other reasons for lower bound convergence problems include poor meshing around stress concentrations or accumulation of numerical error. In contrast to this the upper bound methods such as the Linear Matching Method (Ponter and Chen, 2001a, 2001b), based on Koiter's kinematic theorem, use energy integrals over the whole volume as a convergence criterion. Because the whole model is considered, the localised effects of concentrations become diluted meaning that in general convergence is much quicker and more stable. However, upper bounds do not guarantee conservatism because they will predict shakedown and ratchet loads which are equal to or greater than the least upper bound associated with the class of displacement fields defined by the finite element mesh. Furthermore, recent work by $\mathrm{O}$. Barrera, et al (2009) concerning limit loads calculated by the Linear Matching Method has shown that the convergence of upper bounds calculated by modulus adjustment procedures can appear to have predicted a sub-optimal failure mechanism. Continued solution of the upper bound procedure towards the least upper bound then selects the optimal failure mechanism for the applied loads and boundary conditions. Whist this phenomenon is uncommon in the majority of solutions and does not affect the final converged upper bound limit, the presence of a convergent lower bound can be compared to the upper bound and add confidence to the final converged value.

In order to satisfy the need for conservatism within the nuclear industry, the addition of lower bound calculations to the Linear Matching Method has become an area of interest in recent years. By having shakedown and ratchet assessment procedures which are able to produce both lower and upper bounds simultaneously serves as both a self verification of the implementation and also an indicator of the level of convergence attained. Both lower and upper bounds tending towards a common solution gives confidence in the implementation of the respective bounding theorems, and also mitigates against the "sub-optimal" mechanisms which may be produced by an upper bound solution acting alone. The difference between the two final answers gives clues to the user about how well solutions have converged. Therefore, the Linear Matching Method framework now has shakedown and ratchet assessment procedures which are able to produce both lower and upper bound solutions. 
The initial lower bound shakedown assessment procedure was added to the Linear Matching Method framework by Chen (2010a). This lower bound calculation was performed alongside the upper bound and demonstrated that the lower and upper bounds can produce shakedown limit loads which are within $1 \%$ of each other. Further verification of both lower and upper bounds was provided by application to pipe bends (Chen et al, 2011), welded pipes (Chen et al, 2011a) and composite cylinders (Chen et al, 2011b) where the shakedown bounds were confirmed by full step by step analysis.

More recently, a lower bound to the ratchet calculation was proposed by Ure et al (2011) which used a similar methodology to that of the shakedown procedure. This method has been applied to the analysis of composite materials by Chen (2010b). The upper bound ratchet calculation applies a multiplier to only the constant component of the loading, meaning that the level of the cyclic component of loading is fixed, where the entire cyclic load history is decomposed into a cyclic component and a constant component. However, in the existing lower bound ratchet method (Ure et al, 2011; Chen, 2010b), a scalar multiplier is applied to the entire load history rather than the constant component of the loading. This means that the level of cyclic loading is also scaled. The scaling of the cyclic loading means that the lower bound ratchet limit is effectively being calculated for a different load case, and so cannot be compared to the upper bound in a single calculation. To compare the lower and upper bounds the entire interaction diagram must be created. This is adequate for research purposes, where it is very likely that the entire interaction diagram would be created anyway. In industrial applications to assess the integrity of plant components, however, the level of cyclic loading is fixed and a single calculation is all that is required to determine if the component has sufficient margin against ratcheting.

The purpose of this paper is to propose, explain and demonstrate the addition of a revised lower bound calculation to the Linear Matching Method ratchet analysis procedure. This procedure differs from that proposed in (Ure et al, 2011) in that the multipliers produced in the current paper only apply to the constant component of loading in the same way as the upper bound calculation does, allowing it to be directly compared with the upper bound. In this paper the established upper bound linear matching method is briefly explained, highlighting the details necessary to implement the extended lower bound theorem. Melan's static shakedown theorem is then extended to allow the calculation of the lower bound ratchet limit. The equations used to calculate the lower bound multiplier are derived by making use of the von Mises yield criterion and their numerical implementation is then explained. Finally, the convergence of the method is demonstrated by a benchmark example of a holed plate. The further application and verification of this new lower bound LMM to a practical problem is provided in an accompanying paper (Ure et al, 2012), where a pipe intersection with a dissimilar material weld is subjected to cyclic thermal and mechanical loading.

\section{The Linear Matching Method}

The LMM has been described at great length in other works (Ponter and Chen, 2001a, 2001b) and it would be impractical to fully report the entire method here. Instead a brief explanation will be given highlighting the details necessary to explain the lower bound method, which is the main subject of this paper. 
The linear matching method is an iterative procedure based entirely on linear solutions with spatially varying moduli. At points where the stress is above the yield stress, the modulus is reduced. The next solution in the iterative procedure uses this modified value of modulus, and the stresses redistribute in the same way as they would with an elastic-plastic material.

Consider a body of volume $V$ and surface area $S$. A cyclic temperature history $\theta\left(x_{i}, t\right)$ acts within the volume and varying mechanical loads $\mathrm{P}\left(\mathrm{x}_{\mathrm{i}}, \mathrm{t}\right)$ and constant mechanical loads, $\overline{\mathrm{F}}\left(\mathrm{x}_{\mathrm{i}}\right)$, act on part of the surface $S_{T}$. The remainder of the surface is constrained to have zero displacement rate. These loads act over a time cycle of $0 \leq t \leq \Delta t$, and can be decomposed into their constant and cyclic components:

$$
F\left(x_{i}, t\right)=\lambda \bar{F}\left(x_{i}\right)+\theta\left(x_{i}, t\right)+P\left(x_{i}, t\right)
$$

where $\lambda$ is a load parameter. The linear elastic stress history associated with these loads is:

$$
\hat{\sigma}_{i j}\left(x_{k}, t\right)=\lambda \hat{\sigma}_{i j}^{\bar{F}}\left(x_{k}\right)+\hat{\sigma}_{i j}^{\Delta}\left(x_{k}, t\right) \text { where } \hat{\sigma}_{i j}^{\Delta}\left(x_{k}, t\right)=\hat{\sigma}_{i j}^{P}\left(x_{k}, t\right)+\hat{\sigma}_{i j}^{\theta}\left(x_{k}, t\right)
$$

where $\hat{\sigma}_{i j}^{\bar{F}}, \hat{\sigma}_{i j}^{P}$ and $\hat{\sigma}_{i j}^{\theta}$ represent the stresses due to $\overline{\mathrm{F}}\left(\mathrm{x}_{\mathrm{i}}\right), \mathrm{P}\left(\mathrm{x}_{\mathrm{i}}, \mathrm{t}\right)$ and $\theta\left(\mathrm{x}_{\mathrm{i}}, \mathrm{t}\right)$ respectively. The load parameter $\lambda$ allows a range of loading histories to be considered. For this cyclic problem definition, the stresses and strain rates will asymptotically approach a steady cyclic state where

$$
\sigma_{i j}(t)=\sigma_{i j}(t+\Delta t), \dot{\varepsilon}_{i j}(t)=\dot{\varepsilon}_{i j}(t+\Delta t)
$$

This stress state can be decomposed into four components as shown below in equation (4): the two elastic stresses, $\lambda \hat{\sigma}_{i j}^{\bar{F}}+\hat{\sigma}_{i j}^{\Delta}$, a constant residual stress accumulated up to the beginning of the cycle, $\bar{\rho}_{i j}$, and a residual solution which represents the changes during the cycle, $\rho_{i j}^{r}$,

$$
\sigma_{i j}\left(x_{k}, t\right)=\hat{\sigma}_{i j}^{\Delta}\left(x_{k}, t\right)+\rho_{i j}^{r}\left(x_{k}, t\right)+\lambda \hat{\sigma}_{i j}^{\bar{F}}\left(x_{k}\right)+\bar{\rho}_{i j}\left(x_{k}\right)
$$

The constant residual stress $\bar{\rho}_{i j}$ is the self equilibrating state of stress at the start and end of the cycle caused by with the presence of an additional constant load. For a stable cyclic solution there is no accumulation of stress from one cycle to the next, and therefore:

$$
\rho_{i j}^{r}\left(x_{k}, 0\right)=\rho_{i j}^{r}\left(x_{k}, \Delta t\right)=\bar{\rho}_{i j}^{0}\left(x_{k}\right)
$$

Where $\bar{\rho}_{i j}^{0}\left(x_{k}\right)$ is the constant element of $\rho_{i j}^{r}\left(x_{k}, \Delta t\right)$ associated with the cyclic loading. Based on this stable cyclic formulation, the evaluation of the ratchet limit becomes possible if the applied loading can be decomposed into constant and varying components. Because the structure is subjected to stable cyclic load conditions, the changing residual stress $\rho_{i j}^{r}$ is caused directly by this cyclic load. When stable cyclic loading conditions are established the applied cyclic loading is augmented by the varying residual stress field. The application of a constant load to the component in this stable cyclic state causes the formation of a constant residual stress field $\bar{\rho}_{i j}$. 
With this in mind, the linear matching method is divided into two stages, which are shown in figure 1. The first stage considers only the cyclic loading to evaluate the varying residual stress $\rho_{i j}^{r}$ and the associated plastic strain range. A fixed level of cyclic loading is applied in this stage. The modulus adjustment procedure allows the stresses to redistribute and the varying residual stress field is developed at each point in the load cycle. The second stage then calculates the maximum level of additional constant loading which will not cause the component to ratchet. Stage two is essentially a traditional shakedown assessment to calculate the constant residual stress field where the initial elastic cyclic stress field is augmented by the varying residual stress calculated in stage 1 . This stage calculates the maximum level of additional constant loading (and its associated constant residual stress field) which can be applied to the component subject to this predefined cyclic loading before ratcheting will begin The convergence of stage two is based on Koiter's theorem which states that if 1) any kinematically admissible strain rate can be found such that the strain rate is compatible with the applied displacement and 2) the plastic dissipation within the structure is less than or equal to the applied work, then shakedown does not occur.

At the end of each iteration, the upper bound multiplier $\lambda^{\mathrm{UB}}$ is calculated, which is the level by which the constant loading will be scaled in the next increment. This, combined with the modulus adjustment procedure, produces a series of monotonically reducing upper bounds. A detailed explanation of the implementation of this procedure is given in (Chen and Ponter, 2010).

\section{Extension of Melan's Theorem}

Melan's theorem states that for a given load set the structure will shakedown if a constant residual stress field can be found such that the yield condition is not violated for any combination of cyclic elastic and residual stresses.

$$
f\left(\hat{\sigma}_{i j}\left(x_{k}, t\right)+\bar{\rho}_{i j}\left(x_{k}\right)\right) \leq 0
$$

If the applied cyclic stresses are in excess of strict shakedown then an additional varying residual stress field, $\rho_{i j}^{r}\left(x_{k}, t\right)$, will form and so the total stress at any point in the structure will be given by:

$$
\sigma_{i j}\left(x_{k}, t\right)=\hat{\sigma}_{i j}\left(x_{k}, t\right)+\bar{\rho}_{i j}\left(x_{k}\right)+\rho_{i j}^{r}\left(x_{k}, t\right)
$$

With this additional varying residual stress field it is no longer possible to directly use Melan's theorem as given in equation (6). The primary reason for this is that the stress state of equation (7) may still satisfy the yield condition, but this condition alone does not ensure that ratcheting is not occurring and at present no theorem exists in the literature which extends Melan's theorem to the ratchet limit.

However it is possible to extend Melan's shakedown theorem of equation (6) by careful consideration of the cyclic loading and the nature of the residual stress fields established by these loads. Considering the stress state of equation (7), we use the assumption of Chen and Ponter (2010) that the loading can be decomposed into cyclic and constant components. Therefore for a component subject to predescribed cyclic load conditions we may be able to assess the ratchet limit 
associated with the addition of a further constant load. In doing this the evaluation of the varying residual stress associated with the cyclic loading can be evaluated independently of the constant residual stress field associated with the constant loading.

$$
\hat{\sigma}_{i j}\left(x_{k}, t\right)=\hat{\sigma}_{i j}^{\Delta}\left(x_{k}, t\right)+\lambda \hat{\sigma}_{i j}^{\bar{F}}\left(x_{k}\right)
$$

Where $\hat{\sigma}_{i j}^{\Delta}\left(x_{k}, t\right)$ is the predefined level of time varying applied cyclic stress and $\lambda \hat{\sigma}_{i j}^{\bar{F}}\left(x_{k}\right)$ is the additional time invariant constant applied stress, where $\lambda$ is a scalar multiplier.

Initially the steady state response of the structure to the cyclic loading, $\hat{\sigma}_{i j}^{\Delta}\left(x_{k}, t\right)$, is evaluated which is assumed to be within the ratchet limit. Hence, the application of this loading causes the varying residual stress field to satisfy:

$f\left(\hat{\sigma}_{i j}^{\Delta}\left(x_{k}, t\right)+\rho_{i j}^{r}\left(x_{k}, t\right)\right) \leq 0$

Where $\rho_{i j}^{r}\left(x_{k}, t\right)$ satisfies equation (5), and an associated alternating plasticity mechanism where there is no net accumulation of total strain during the cycle:

$\varepsilon_{i j}^{T}\left(x_{k}, 0\right)=\varepsilon_{i j}^{T}\left(x_{k}, \Delta t\right)$

With the stabilised response of the structure to the cyclic loads and the alternating plasticity mechanism determined, a second stage may be constructed to evaluate the ratchet limit of the structure subject to an additional constant load using an extended version of Melan's theorem. In this extended version of the theorem, the applied cyclic stress, $\hat{\sigma}_{i j}\left(x_{k}, t\right)$ in equation (6), is augmented by the varying residual stress, $\rho_{i j}^{r}\left(x_{k}, t\right)$ from equation (9):

$\sigma_{i j}^{V}\left(x_{k}, t\right)=\hat{\sigma}_{i j}^{\Delta}\left(x_{k}, t\right)+\rho_{i j}^{r}\left(x_{k}, t\right)$

With this augmented cyclic loading the ratchet limit of the component subject to an additional constant load can be assessed if a constant residual stress field can be found such that the yield condition is satisfied everywhere in the structure at all load instances:

$$
f\left(\sigma_{i j}^{V}\left(x_{k}, t\right)+\lambda \hat{\sigma}_{i j}^{\bar{F}}\left(x_{k}\right)+\bar{\rho}_{i j}\left(x_{k}\right)\right) \leq 0
$$

With the loading described here, which is in excess of strict shakedown, this extension to Melan's theorem is capable of finding the lower bound ratchet limit if the level of cyclic loading is assumed to be predefined.

\section{Calculation of the Lower Bound Ratchet Limit}

The upper bound procedure calculates the maximum level of constant loading that will not cause the component to ratchet. The extended version of Melan's theorem derived in section 3 allows a lower bound calculation to accompany this. The lower bound method presented here only scales the 
additional constant loading (which differs from that presented in (Ure et al, 2011) where the entire load history is scaled) by using equation (13) below:

$$
f\left(\sigma_{i j}^{V}\left(x_{k}, t\right)+\lambda^{L B} \hat{\sigma}_{i j}^{\bar{F}}\left(x_{k}\right)+\bar{\rho}_{i j}\left(x_{k}\right)\right) \leq 0
$$

Where $f()$ is the yield function, which in this case is the von-Mises criterion. Since we are interested in determining the level of additional constant loading which will not cause ratcheting, $\lambda^{\mathrm{LB}}$ is used as the scalar multiplier to determine this. If the component is not ratcheting then it should be possible to scale the elastic constant load in order to satisfy the yield condition for all points in the load cycle and at all locations in the structure.

This lower bound process can be implemented in the linear matching method as an additional calculation to complement the separate upper bound method. The linear matching method is fundamentally an upper bound method and so the convergence of calculations performed will still be based on the kinematic theorem of Chen and Ponter (2010). During each increment in the solution, the residual stress fields calculated by the upper bound method can be used in equation (13) to evaluate $\lambda^{\mathrm{LB}}$, and so gives the level by which the additional constant loading must be scaled in order to make the total effective stress equal to the yield stress. When the modulus adjustment procedure has allowed the stresses to redistribute, the lower and upper bound multipliers calculated in each increment should converge towards the same solution.

In a three dimensional stress state each of the three stresses at that point in the load cycle (additional constant, constant residual and total varying stresses) has six components, which makes the stress state at any point in the model a function of eighteen stress components. These eighteen stress components and the yield stress are all known from the upper bound calculation, which leaves the multiplier, $\lambda^{\mathrm{LB}}$, as the only unknown. Equation (13) can be re-arranged to find this value. The algebra involved in the re-arrangement for $\lambda^{\mathrm{LB}}$ is cumbersome and so is detailed in the appendix, but results in a quadratic equation:

$0=A\left(\lambda^{L B}\right)^{2}+B \lambda^{L B}+C$

where A, B and C are coefficients containing all 18 stress coefficients and the yield stress. Solution of this quadratic is easily performed using the quadratic formula:

$$
\lambda^{L B}=\frac{-B \pm \sqrt{B^{2}-4 A C}}{2 A}
$$

This gives the lower bound multiplier for that point in the structure and at one extreme in the load cycle. This equation can be used for all extremes in the load cycle to give the lower bound multipliers which would satisfy yield at those load conditions. The lowest value of $\lambda^{\mathrm{LB}}$ out of all the load extremes is taken as the value for that point. Performing this procedure over the entire structure results in every point in the structure having an associated value of $\lambda^{\mathrm{LB}}$ which will satisfy yield at all points in the load cycle. Using the minimum $\lambda^{\mathrm{LB}}$ value from every point in the structure gives the lower bound multiplier for the entire structure - the scalar value which if used to scale the 
additional constant loading will mean that all points in the structure will have stresses which satisfy the yield condition at all points in the load cycle.

\section{Numerical Implementation}

The Linear Matching Method is implemented in Abaqus finite element analysis software (Abaqus, 2009) by using a UMAT subroutine. As described in Chen and Ponter (2010), the first stage of the calculation process considers the cyclic loading and establishes the stabilised cycle (namely $\hat{\sigma}_{i j}^{\Delta}\left(x_{k}, t\right)+\rho_{i j}^{r}\left(x_{k}, t\right)$ in equation 11). During stage 1 , the stresses at each point in the load cycle are evaluated based on the input elastic stresses and the varying residual stress field is established. When stage 1 has completed, stage 2 then uses this varying residual stress field to calculate the upper bound ratchet limit by scaling the additional constant loading. During this upper bound calculation procedure, the constant residual stress field $\bar{\rho}_{i j}\left(x_{k}\right)$ is calculated as part of the procedure.

Numerically, all of these calculations occur at integration point level during both stage 1 and 2 . The subroutine considers each integration point individually: the modulus is modified, and the new levels of stress and plastic strain are calculated along with the energies required for the upper bound solution. The upper bound multiplier, $\lambda^{\mathrm{UB}}$, is calculated at the end of the increment by integrating the energies calculated over the volume of the structure.

The lower bound calculation procedure described in section 4 is performed alongside the upper bound calculations during stage 2 of the solution process. The upper bound calculation adjusts the modulus of the integration point and then uses this to calculate updated values for the constant residual stress field. Along with the elastic stresses from the applied constant loading and the total varying stress from stage 1 , the upper bound calculation has then defined all the stresses that define the stress state at the point. At this stage, the lower bound extracts the 18 stress components needed to calculate the coefficients $A, B$ and $C$ of the quadratic of equation (14). This quadratic can then be solved to find $\lambda^{\mathrm{LB}}$ for that point and load instance. That is the multiplier, which if applied to the constant part of the applied stress at that point, would make the effective stress at that point and load instance equal to or less than the yield stress.

In reality, the numerical nature of the solution procedure means that the solution to the quadratic has three possible outcomes which are shown graphically in Fig. 2 . The horizontal $\lambda^{\mathrm{LB}}$ axis crosses the stress axis at the yield stress. Any values below this are therefore below the yield stress, and values above are above yield.

The response in Fig. 2a) is the most common, where there are two real and distinct roots. In terms of the stresses, this means that any value of $\lambda^{\mathrm{LB}}$ which was between these two roots would bring the total effective stress at that point below yield. For the solution for the ratchet limit, the maximum positive root is taken, as this is the maximum level of additional constant loading which will not cause ratcheting.

The second possible response is that there is only one root (Fig. 2b). Physically this means that only multiplying the additional constant stress by that number will bring the stress down to equal the yield stress. Being a numerical simulation, it is rare that an integration point will only have one root. 
The third possibility is that the integration point has no roots at all, meaning that it is impossible to bring the stress to below yield by scaling only the additional constant load (Fig. 2c). Theoretically, if the component is not ratcheting, this scenario should not occur. This situation arises due the accumulation of numerical errors and slight inaccuracies during the solution process. In reality the stress may only be fractions of a percent above yield (and so to the user is effectively equal to yield) but in terms of the quadratic equation this is enough to prevent solution. When this situation arises, the stress components at the point are stored for later validation of $\lambda^{\mathrm{LB}}$.

To obtain the lower bound multiplier for the entire model the following procedure is used:

1. Use equation (15) to calculate $\lambda^{\mathrm{LB}}$ at each integration point and at each load instance.

2. Take the average of these values over each element for each load instance. That is, for each load instance, the values of $\lambda^{\mathrm{LB}}$ at the integration points are averaged within the element.

3. The minimum of all of the element averages for all load instances is taken as the lower bound multiplier for the model.

Elemental averages of $\lambda^{\mathrm{LB}}$ are taken to mitigate against the convergence problems which can occur due to stress concentrations and discontinuities. It is possible, by accumulation of numerical error, that some integration points in critical regions may produce disproportionately low $\lambda^{\mathrm{LB}}$ values compared to the other points around it. If the minimum value in the model was taken to be the overall multiplier then this single integration point would be preventing convergence simply because of numerical errors. Averaging all the $\lambda^{\mathrm{LB}}$ values over the element is a safeguard against this by diluting the effect of any single integration point.

When the lower bound multiplier for the model is known, the points which had no roots to the quadratic are re-evaluated. The effective stress at these points is re-calculated using the lower bound multiplier of the model, and if it is found to be above a threshold ( $5 \%$ above yield, for example) then a warning is given to the user. The number of points above this threshold is a useful indicator of the level of stress redistribution achieved by the modulus adjustment procedure, and so gives the user a useful measure of the level of convergence.

\section{Numerical Verification - Plate with Central Hole}

The method described has been applied to simple numerical example of a plate with a central hole in order to demonstrate the solution and convergence properties.

\section{$\underline{6.1 \text { Problem Description }}$}

Fig. 3 shows the geometry and finite element mesh used for the holed plate. The ratio between the diameter of the hole $D$ and the length of the plate $L$ is 0.2 . The ratio between the thickness $T$ and $L$ is 0.05. Due to the symmetry of the geometry and loading, a quarter model is used with the appropriate boundary conditions. In addition, the free edges of the plate are constrained to expand in-plane to simulate the expansion of a large plate. The geometry is meshed with 642 elements of type C3D20R, a quadratic brick element with reduced integration.

The plate is subject to a cyclic temperature gradient between the bore of the hole and the outer edges. The temperature distribution as a function of radius is given in equation (16), which gives a 
temperature difference between the bore of the hole and the edge of the plate of $\Delta \theta$. This distribution approximates to a temperature of $\theta=\bar{\theta}(\mathrm{t})$ at the bore of the hole and $\theta_{0}$ at the edge of the plate.

$\theta(r, t)=\theta_{0}+\left(\bar{\theta}(t)-\theta_{0}\right) \ln (5 a / r) / \ln (5)$

Fig $3 c$ shows the temperature history around the edge of the hole resulting in the two load extremes used in the analysis. In addition to this cyclic thermal loading, a constant uniaxial tension, $\mathrm{P}$, is applied along one edge of the plate. The plate material is assumed to be elastic-perfectly plastic with a yield stress of $360 \mathrm{MPa}$ and a thermal expansion coefficient of $5 \times 10^{-5}{ }^{\circ} \mathrm{C}^{-1}$.

\section{$\underline{6.2 \text { Results }}$}

The ratchet interaction diagram for the plate is shown in Fig. 4 where the applied temperature difference $\Delta \theta$ is normalised against the reference temperature difference of $\Delta \theta_{0}=100^{\circ} \mathrm{C}$ and the uniaxial tension $P$ is normalised against the yield stress of the material $\sigma_{y}=360 \mathrm{MPa}$.

The alternating plasticity limit, calculated by the linear matching method shakedown procedure, is also shown in the figure and thus shows the capability of the linear matching method to calculate lower and upper bound shakedown and ratchet limits.

The interaction diagram follows the classic bree-like shape, with lower and upper bound converging very closely. The convergence of the lower and upper bounds at points $A$ and $B$ is shown in figure 5 .

It is thought that the overall convergence is good in both cases with lower and upper bounds converging to a common solution within around 70 increments. The speedy convergence of the lower bound is owed in part to the fact that the lower bound is calculated at an elemental level. It is thought that this smoothing process is a necessary and important addition to the lower bound calculation procedure. Whilst this approximation reduces the accuracy of the model, the user must bear in mind that the lower and upper bounds are there to complement and validate each other. Without this averaging, the user would need to run the model for many more increments (in some cases three or four times as many) for a lower bound solution taken at integration point level to obtain the same multiplier. It is thought that this version of the lower bound gives a better speed of solution, which is still accurate enough if the user has a sufficient element density in regions of interest.

In both cases the upper bound converges more quickly than the lower bound. This is due to the fact that the upper bound integrates energies over the volume, diluting the effect of the stress concentration at the hole. The lower bound requires a greater number of iterations for the modulus adjustment procedure to redistribute the stress and therefore satisfy the extended Melan's theorem. The convergence plot also shows that the lower bound at point $B$ (within strict shakedown) converges more quickly than point $A$ (which is global shakedown). This is due to the fact that point $B$ has no varying residual stress and thus a simpler stress state. Stage 1 of the calculation is still performed but converges almost instantly and moves onto stage 2 . Point $A$, however, will have a significant level of varying residual stress and so will require more stage 1 increments to allow the stress to redistribute. The build up of numerical error with these additional increments and the fact that the stress fields are more complex in the first place means that the lower bound requires longer 
to satisfy yield at all points in the model. The plateaus seen in the convergence of point $A$ are a result of the subroutine using the "best" value of lower bound calculated up to that increment. When the stress redistribution is taking place it is possible for the stress distributions to produce a worse lower bound than in the previous increment (especially within the first few increments when the rates of change of modulus and stress are high). As a result, the subroutines are programmed to use the best value of lower bound calculated up to that point.

\section{Conclusion}

In this paper an extension to Melan's lower bound shakedown theorem has been proposed for structures subject to cyclic loading which is greater than the shakedown limit. This allows the calculation of the ratchet limit, which is of use to the nuclear industry where components are allowed to be in global shakedown. This extended theorem has been implemented into the Linear matching method ratchet limit calculation procedure. Using a re-arrangement of the von-Mises yield equation, a multiplier is calculated which scales the additional constant loading so that, for a given level of cyclic loading, the component will not ratchet. This method has been applied to the case of a plate with a central hole so that convergence properties may be explored, and it can be seen that accurate lower bounds are obtained.

\section{Acknowledgements}

The authors gratefully acknowledge the support of the Engineering and Physical Sciences Research Council of the United Kingdom, The University of Strathclyde and EDF Energy during the course of this work.

\section{$\underline{\text { References }}$}

Abaqus, 2009. User's Manual Version 6.9.

Ainsworth R.A. (editor), 2003. R5: an assessment procedure for the high temperature response of structures. British Energy Generation Ltd.

Barrera O., Cocks A.F.C., Ponter A.R.S, 2009. Evaluation of the Convergent Properties of the Linear Matching Method for Computing the Collapse of Structural Components. European Journal of Mechanics - A/Solids, 28, 655-667.

Bocciarelli M., Cocchetti G., Maier G., 2004. Shakedown Analysis of Train Wheels by Fourier Series and Nonlinear Programming. Engineering Structures, 26, 455-470.

Chen H.F., 2010a. Lower and upper bound shakedown analysis of structures with temperaturedependent yield stress. ASME Journal of Pressure Vessel Technology, 132(1):011202 (8 pages).

Chen H.F., 2010b. Linear Matching Method for Design Limits in Plasticity. Computers, Materials \& Continua, 20(2), 159-183.

Chen H.F., Ponter A.R.S., 2010. A Direct Method on the Evaluation of Ratchet Limit. Journal of Pressure Vessel Technology, 132(4), 041202 (8 pages).

Chen H.F., Li T., Chen W., Ure J., 2011a. On the shakedown analysis of welded pipes. International Journal of Pressure Vessels and Piping, 88, 301-310. 
Chen H.F., Chen W., Li T., Ure J., 2011b. Shakedown analysis of a composite cylinder with a cross hole. ASME Journal of Pressure Vessel Technology, 133(3):031026 (9 pages).

Chen H.F., Ure J., Li T., Chen W., MacKenzie D., 2011. Shakedown and limit analysis of $90^{\circ}$ pipe bends under internal pressure, cyclic in-plane bending and cyclic thermal loading. International Journal of Pressure Vessels and Piping, 88, 213-222.

Hamilton R., Muscat M., Boyle J.T., 2002. Shakedown analysis for Complex loading using superposition. J. Strain Analysis 37(5), 399-411

Koiter W.T., 1960. General theorems for elastic plastic solids, in: Sneddon J.N., Hill R. (Eds.), Progress in solid mechanics. North Holland, Amsterdam, pp. 167-221.

Liu Y.H., Carvelli V., Maier G., 1997. Integrity assessment of defective pressurized pipelines by direct simplified methods. International Journal of Pressure Vessels and Piping, 74, 49-57.

Mackenzie D., Boyle J.T., Hamilton R., 2000. The elastic compensation method for limit and shakedown analysis: a review. Trans IMechE, Journal of Strain Analysis for Engineering Design, 35(3), 171-188.

Melan E., 1936. Theorie statisch unbestimmter systeme aus ideal-plastichem baustoff. Sitzungsber. d. Akad. d. Wiss. Wien 2A(145), 195-218.

Ponter A.R.S., Chen H.F., 2001a. A minimum theorem for cyclic load in excess of shakedown, with application to the evaluation of a ratchet limit. European Journal of Mechanics - A/Solids, 20(4), 539553.

Ponter A.R.S., Chen H.F., 2001b. A method for the evaluation of a ratchet limit and the amplitude of plastic strain for bodies subjected to cyclic loading. European Journal of Mechanics - A/Solids, 20(4), 555-571.

Seshadri R., 1995. Inelastic evaluation of mechanical and structural components using the generalized local stress strain method of analysis. Nuclear Engineering and Design, 153(2-3) 287-303.

Simon J.W., Weichart D., 2011. Numerical lower bound shakedown analysis of engineering structures. Computer Methods in Applied Mechanics and Engineering, 200, 2828-2839.

Staat, M., Heitzer, M. (Eds.), 2003. Numerical Methods for Limit and Shakedown Analysis . NICSeries, vol. 15. John von Neumann Institute for Computing, Jülich.

Ure J., Chen H.F., Li T., Chen W., Tipping D., MacKenzie D., 2011. A direct method for the evaluation of lower and upper ratchet limits. Procedia Engineering, 10, 356-361.

Ure J., Chen H., Tipping D., 2012. Calculation of a Lower Bound Ratchet Limit Part 2 - Application to a Pipe Intersection with Dissimilar Material Join. European Journal of Mechanics - A/Solids, (volume), (Page Numbers). 


\section{List of Figures}

Figure 1 - Two stages of the Linear Matching Method ratchet analysis

Figure 2 - Possible solutions to equation (15)

Figure 3 - Holed plate a) geometry, b) mesh and c) cyclic load history

Figure 4 - Holed plate shakedown and ratchet interaction diagram

Figure 5 - Convergence of Points $A$ and $B$ 


\section{Appendix}

Equation A.1 defines the total effective stress at any point in the finite element model.

$$
\sigma_{i j}\left(x_{k}, t\right)=\sigma_{i j}^{V}\left(x_{k}, t\right)+\lambda^{L B} \hat{\sigma}_{i j}^{\bar{F}}\left(x_{k}\right)+\bar{\rho}_{i j}\left(x_{k}\right)
$$

And in order to satisfy the extended Melan's theorem, this stress state must satisfy the yield condition, according to equation A.2.

$$
f\left(\sigma_{i j}^{V}\left(x_{k}, t\right)+\lambda^{L B} \hat{\sigma}_{i j}^{\bar{F}}\left(x_{k}\right)+\bar{\rho}_{i j}\left(x_{k}\right)\right) \leq 0
$$

In the context of the linear matching method, we consider an elastic-perfectly plastic material which satisfies the von-Mises yield criterion. This then means:

$$
f\left(\sigma_{i j}^{V}\left(x_{k}, t\right)+\lambda^{L B} \hat{\sigma}_{i j}^{\bar{F}}\left(x_{k}\right)+\bar{\rho}_{i j}\left(x_{k}\right)\right) \leq \sigma_{y}
$$

Where $\sigma_{y}$ is the yield stress. During the incremental solution procedure the eighteen stress components and the yield stress are known values for each point in the load cycle. Therefore, to calculate the lower bound multiplier for an integration point at one point in the load cycle, the equation for the von-Mises yield stress can be expanded and re-arranged:

$$
\begin{aligned}
\frac{2}{3} \sigma_{y i e l d}^{2}= & {\left[\left(\lambda^{L B} \hat{\sigma}_{x}^{\bar{F}}+\bar{\rho}_{x}+\sigma_{x}^{V}\right)-\left(\frac{\left(\lambda^{L B} \hat{\sigma}_{x}^{\bar{F}}+\bar{\rho}_{x}+\sigma_{x}^{V}\right)+\left(\lambda^{L B} \hat{\sigma}_{y}^{\bar{F}}+\bar{\rho}_{y}+\sigma_{y}^{V}\right)+\left(\lambda^{L B} \hat{\sigma}_{z}^{\bar{F}}+\bar{\rho}_{z}+\sigma_{z}^{V}\right)}{3}\right)\right]^{2} } \\
& +\left[\left(\lambda^{L B} \hat{\sigma}_{y}^{\bar{F}}+\bar{\rho}_{y}+\sigma_{y}^{V}\right)-\left(\frac{\left(\lambda^{L B} \hat{\sigma}_{x}^{\bar{F}}+\bar{\rho}_{x}+\sigma_{x}^{V}\right)+\left(\lambda^{L B} \hat{\sigma}_{y}^{\bar{F}}+\bar{\rho}_{y}+\sigma_{y}^{V}\right)+\left(\lambda^{L B} \hat{\sigma}_{z}^{\bar{F}}+\bar{\rho}_{z}+\sigma_{z}^{V}\right)}{3}\right)\right]^{2} \\
& +\left[\left(\lambda^{L B} \hat{\sigma}_{z}^{\bar{F}}+\bar{\rho}_{z}+\sigma_{z}^{V}\right)-\left(\frac{\left(\lambda^{L B} \hat{\sigma}_{x}^{\bar{F}}+\bar{\rho}_{x}+\sigma_{x}^{V}\right)+\left(\lambda^{L B} \hat{\sigma}_{y}^{\bar{F}}+\bar{\rho}_{y}+\sigma_{y}^{V}\right)+\left(\lambda^{L B} \hat{\sigma}_{z}^{\bar{F}}+\bar{\rho}_{z}+\sigma_{z}^{V}\right)}{3}\right)\right]^{2} \\
& +\left(\lambda^{L B} \hat{\sigma}_{x y}^{\bar{F}}+\bar{\rho}_{x y}+\sigma_{x y}^{V}\right)^{2}+\left(\lambda^{L B} \hat{\sigma}_{y z}^{\bar{F}}+\bar{\rho}_{y z}+\sigma_{y z}^{V}\right)^{2}+\left(\lambda^{L B} \hat{\sigma}_{x z}^{\bar{F}}+\bar{\rho}_{x z}+\sigma_{x z}^{V}\right)^{2}
\end{aligned}
$$

Where $\frac{\left(\lambda^{L B} \hat{\sigma}_{x}^{\bar{F}}+\bar{\rho}_{x}+\sigma_{x}^{V}\right)+\left(\lambda^{L B} \hat{\sigma}_{y}^{\bar{F}}+\bar{\rho}_{y}+\sigma_{y}^{V}\right)+\left(\lambda^{L B} \hat{\sigma}_{z}^{\bar{F}}+\bar{\rho}_{z}+\sigma_{z}^{V}\right)}{3}$ is the hydrostatic stress.

Re-arranging this for $\lambda^{\mathrm{LB}}$ gives a quadratic:

$0=A\left(\lambda^{L B}\right)^{2}+B \lambda^{L B}+C$

With coefficients $A, B$ and $C$ being: 


$$
\begin{aligned}
& A=-\frac{2}{3} \hat{\sigma}_{x}^{\bar{F}} \hat{\sigma}_{y}^{\bar{F}}+2\left(\hat{\sigma}_{x y}^{\bar{F}}\right)^{2}+\frac{2}{3}\left(\hat{\sigma}_{x}^{\bar{F}}\right)^{2}+2\left(\hat{\sigma}_{x z}^{\bar{F}}\right)^{2}-\frac{2}{3} \hat{\sigma}_{x}^{\bar{F}} \hat{\sigma}_{z}^{\bar{F}} \\
& +\frac{2}{3}\left(\hat{\sigma}_{y}^{\bar{F}}\right)^{2}+\frac{2}{3}\left(\hat{\sigma}_{z}^{\bar{F}}\right)^{2}+\frac{2}{3} \hat{\sigma}_{y}^{\bar{F}} \hat{\sigma}_{z}^{\bar{F}}+\frac{2}{3}\left(\hat{\sigma}_{y z}^{\bar{F}}\right)^{2} \\
& B=-\frac{2}{3} \bar{\rho}_{y} \hat{\sigma}_{z}^{\bar{F}}+4 \hat{\sigma}_{y z}^{\bar{F}} \bar{\rho}_{y z}+4 \hat{\sigma}_{x z}^{\bar{F}} \sigma_{x z}^{V}-\frac{2}{3} \hat{\sigma}_{x}^{\bar{F}} \bar{\rho}_{y} \\
& +\frac{4}{3} \hat{\sigma}_{y}^{\bar{F}} \bar{\rho}_{y}+4 \hat{\sigma}_{x y}^{\bar{F}} \sigma_{x y}^{V}-\frac{2}{3} \sigma_{x}^{V} \hat{\sigma}_{y}^{\bar{F}}+\frac{4}{3} \hat{\sigma}_{z}^{\bar{F}} \sigma_{z}^{V} \\
& +4 \hat{\sigma}_{x z}^{\bar{F}} \bar{\rho}_{x z}-\frac{2}{3} \hat{\sigma}_{x}^{\bar{F}} \sigma_{y}^{V}+\frac{4}{3} \hat{\sigma}_{z}^{\bar{F}} \bar{\rho}_{z}+\frac{4}{3} \hat{\sigma}_{x}^{\bar{F}} \sigma_{x}^{V} \\
& +\frac{4}{3} \hat{\sigma}_{y}^{\bar{F}} \sigma_{y}^{V}-\frac{2}{3} \bar{\rho}_{x} \hat{\sigma}_{z}^{\bar{F}}+\frac{4}{3} \hat{\sigma}_{x}^{\bar{F}} \bar{\rho}_{x}-\frac{2}{3} \sigma_{y}^{V} \hat{\sigma}_{z}^{\bar{F}} \\
& -\frac{2}{3} \bar{\rho}_{x} \hat{\sigma}_{y}^{\bar{F}}+4 \hat{\sigma}_{x y}^{\bar{F}} \bar{\rho}_{x y}-\frac{2}{3} \sigma_{x}^{V} \hat{\sigma}_{z}^{\bar{F}}+4 \hat{\sigma}_{y z}^{\bar{F}} \sigma_{y z}^{V} \\
& -\frac{2}{3} \hat{\sigma}_{y}^{\bar{F}} \bar{\rho}_{z}-\frac{2}{3} \hat{\sigma}_{x}^{\bar{F}} \sigma_{z}^{V}-\frac{2}{3} \hat{\sigma}_{y}^{\bar{F}} \sigma_{z}^{V}-\frac{2}{3} \hat{\sigma}_{x}^{\bar{F}} \bar{\rho}_{z} \\
& C=2\left(\sigma_{y z}^{V}\right)^{2}-\frac{2}{3} \sigma_{y}^{V} \bar{\rho}_{z}+\frac{2}{3}\left(\bar{\rho}_{x}\right)^{2}+\frac{2}{3}\left(\bar{\rho}_{z}\right)^{2}+2\left(\sigma_{x z}^{V}\right)^{2}-\frac{2}{3} \bar{\rho}_{y} \sigma_{z}^{V} \\
& -\frac{2}{3} \bar{\rho}_{y} \bar{\rho}_{z}+4 \sigma_{x y}^{V} \bar{\rho}_{x y}-\frac{2}{3} \sigma_{x}^{V} \sigma_{z}^{V}-\frac{2}{3} \sigma_{x}^{V} \bar{\rho}_{z} \\
& -\frac{2}{3} \bar{\rho}_{x} \sigma_{z}^{V}-\frac{2}{3} \bar{\rho}_{x} \bar{\rho}_{z}+\frac{4}{3} \sigma_{z}^{V} \bar{\rho}_{z}+2\left(\sigma_{z}^{V}\right)^{2}+2\left(\bar{\rho}_{x y}\right)^{2} \\
& +2\left(\bar{\rho}_{x z}\right)^{2}+2\left(\sigma_{x y}^{V}\right)^{2}-\frac{2}{3} \bar{\rho}_{x} \sigma_{y}^{V}-\frac{2}{3} \sigma_{x}^{V} \sigma_{y}^{V}-\frac{2}{3} \bar{\rho}_{x} \bar{\rho}_{y} \\
& +4 \sigma_{x z}^{V} \bar{\rho}_{x z}+\frac{4}{3} \sigma_{y}^{V} \bar{\rho}_{y}-\frac{2}{3} \sigma_{x}^{V} \bar{\rho}_{y}+\frac{2}{3}\left(\sigma_{x}^{V}\right)^{2}+\frac{2}{3}\left(\sigma_{y}^{V}\right)^{2} \\
& +\frac{4}{3} \sigma_{x}^{V} \bar{\rho}_{x}-\frac{2}{3} \sigma_{y}^{V} \sigma_{z}^{V}+\frac{2}{3}\left(\bar{\rho}_{y z}\right)^{2}+\frac{4}{3} \sigma_{y z}^{V} \bar{\rho}_{y z}+\frac{2}{3}\left(\bar{\rho}_{y}\right)^{2} \\
& -\frac{2}{3}\left(\sigma_{y}\right)^{2}
\end{aligned}
$$




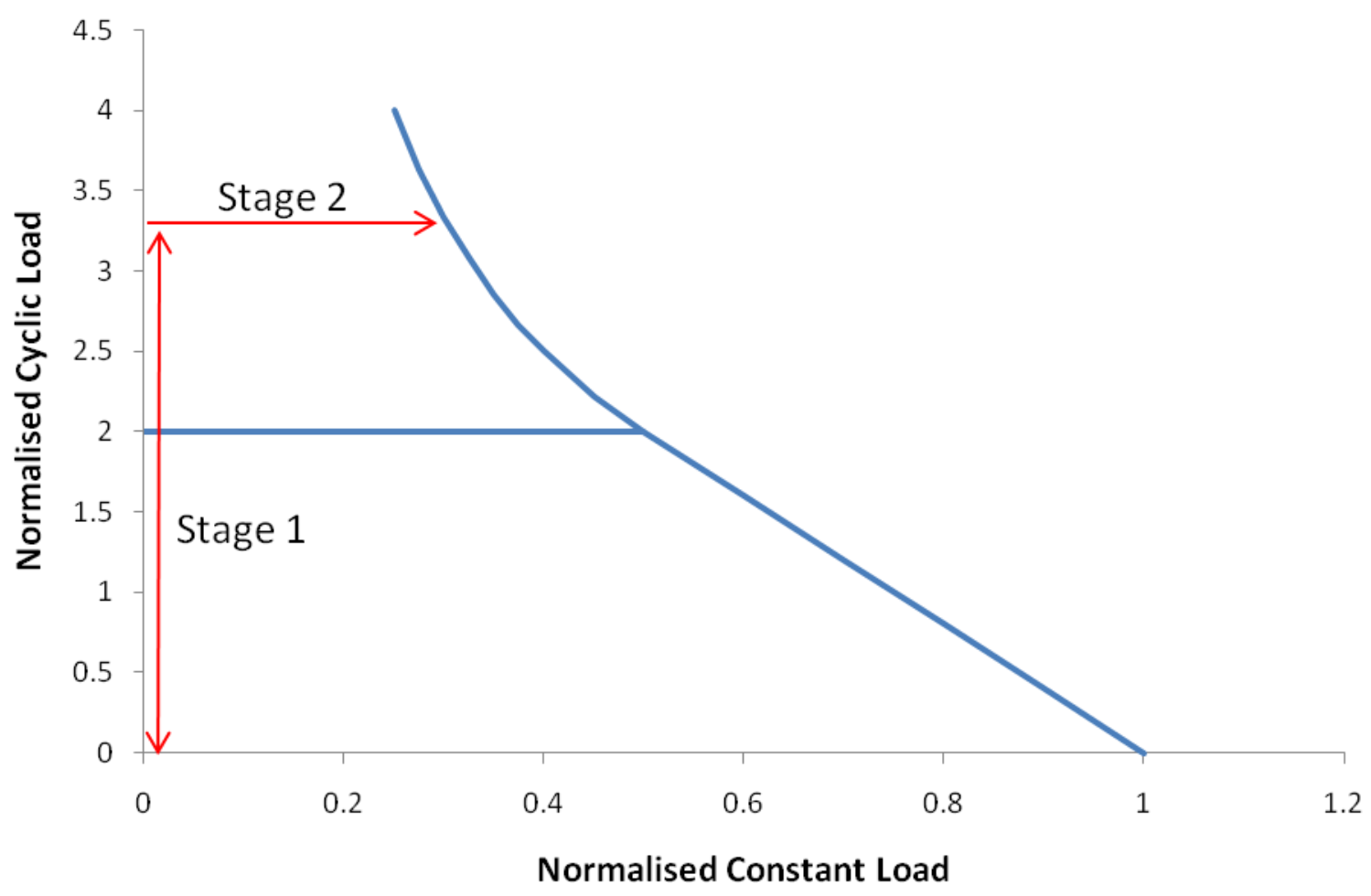

Figure 1 - Two stages of the Linear Matching Method ratchet analysis 

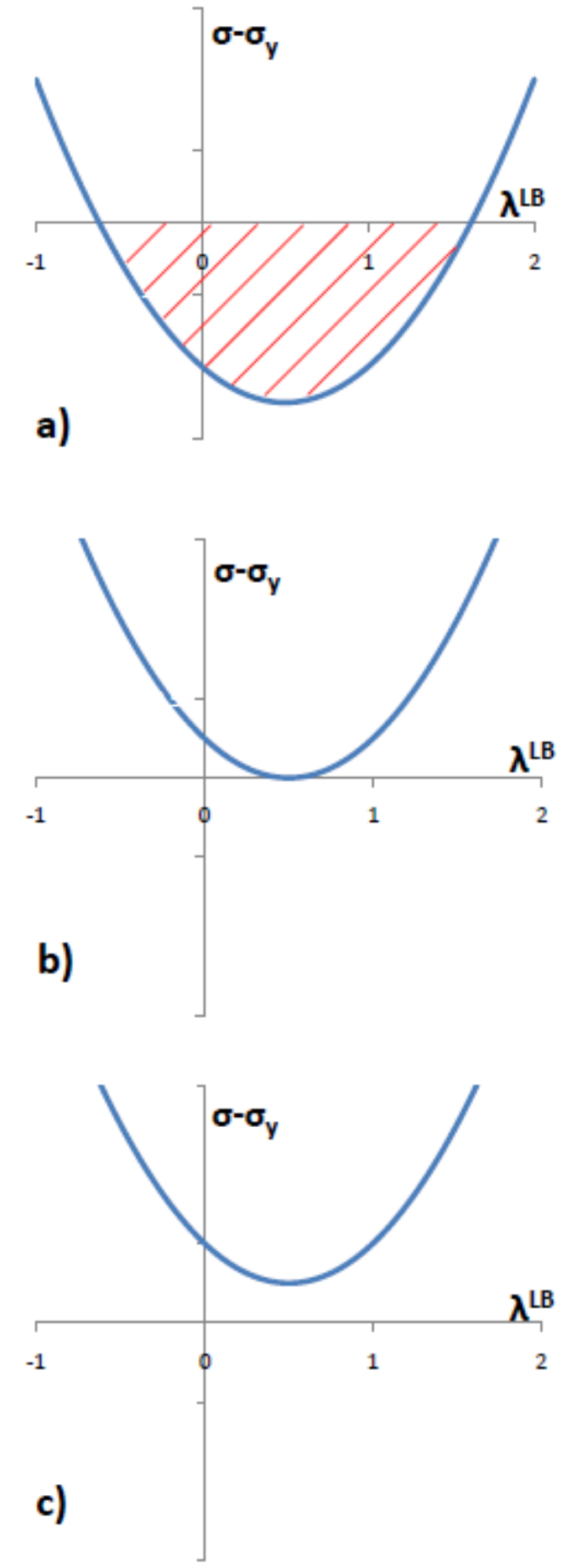

Figure 2 - Possible solutions to equation (15) 


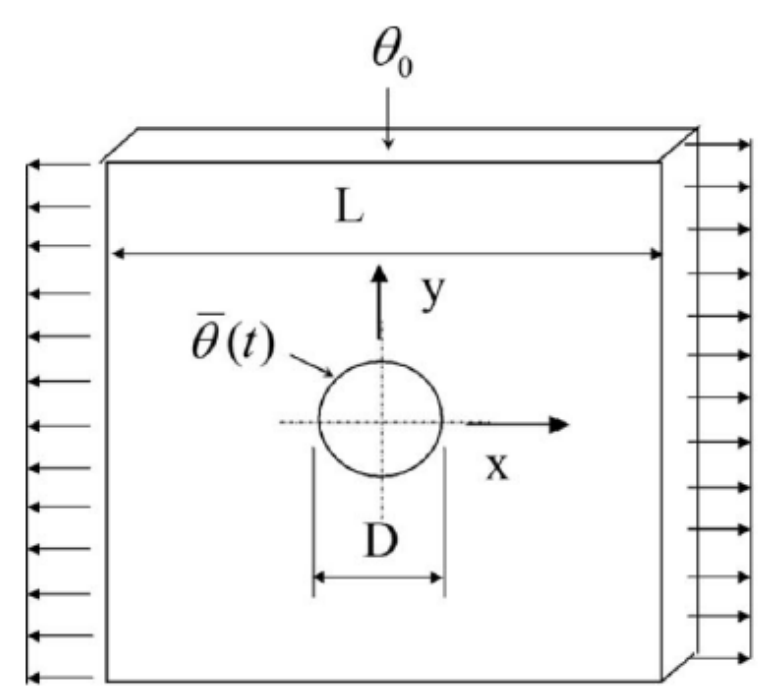

a)

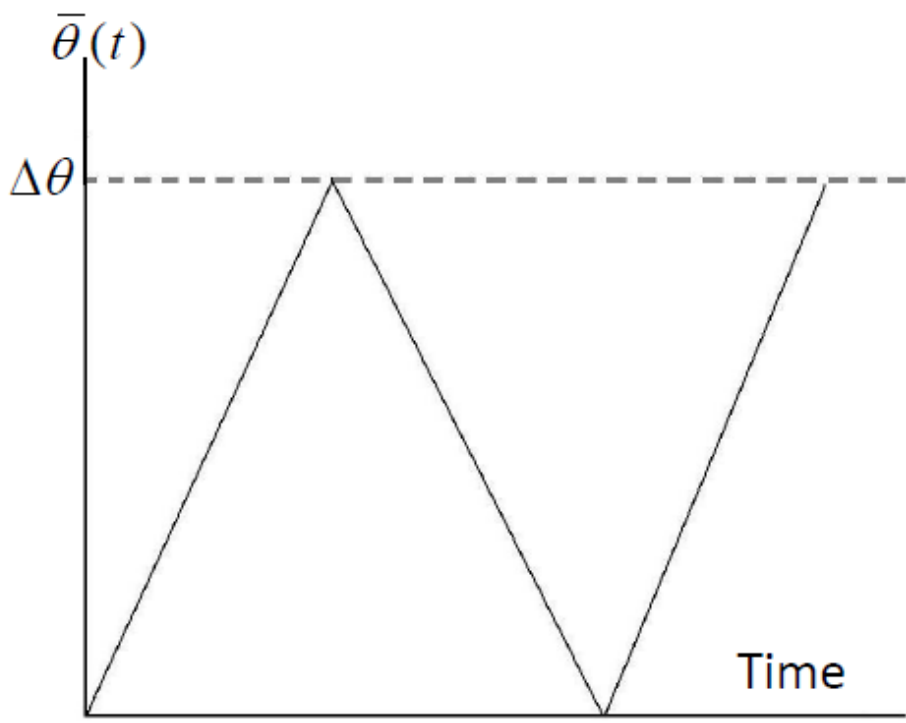

c)

Figure 3 - Holed plate a) geometry, b) mesh and c) cyclic load history 


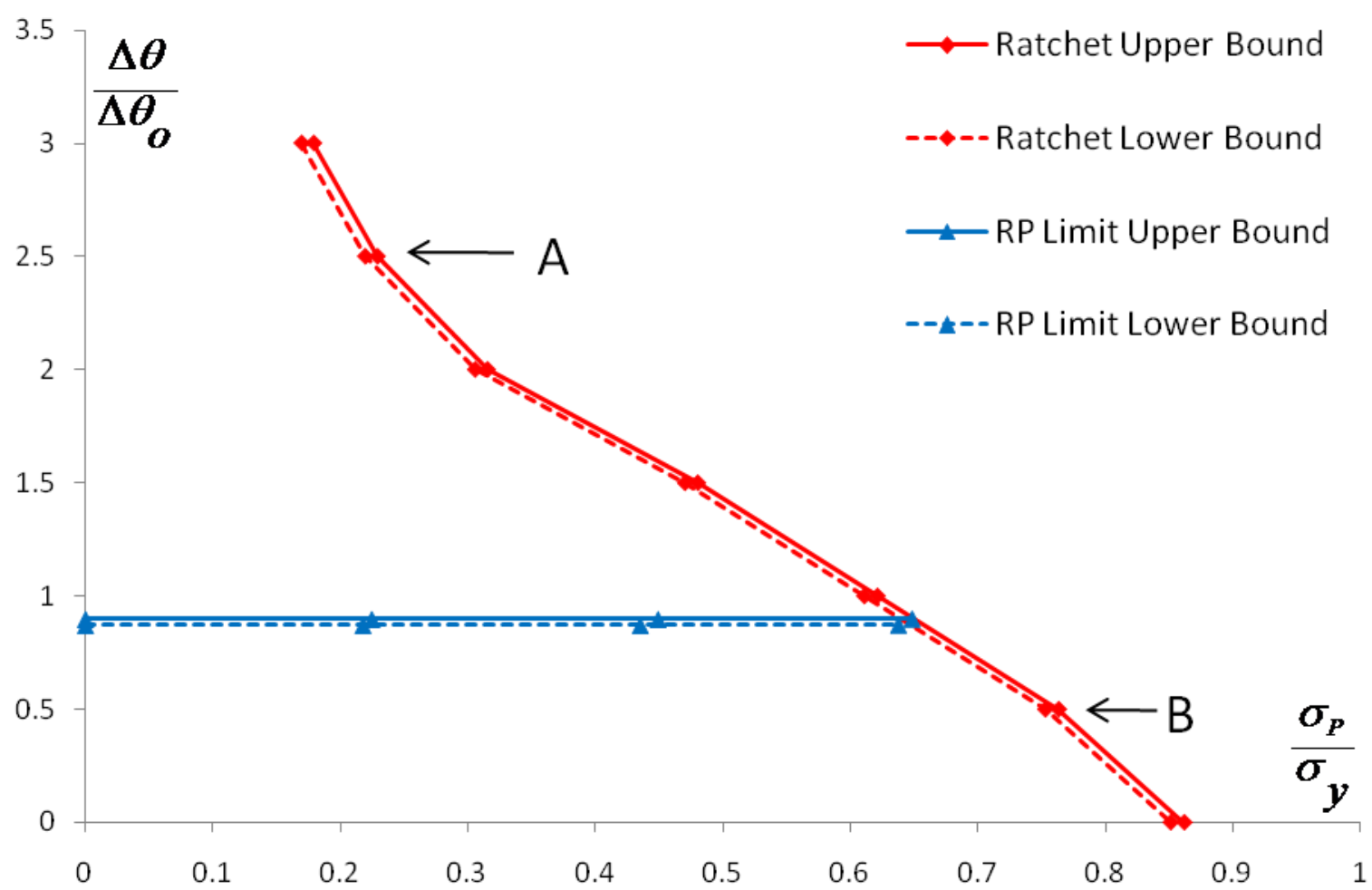

Figure 4 - Holed plate shakedown and ratchet interaction diagram 


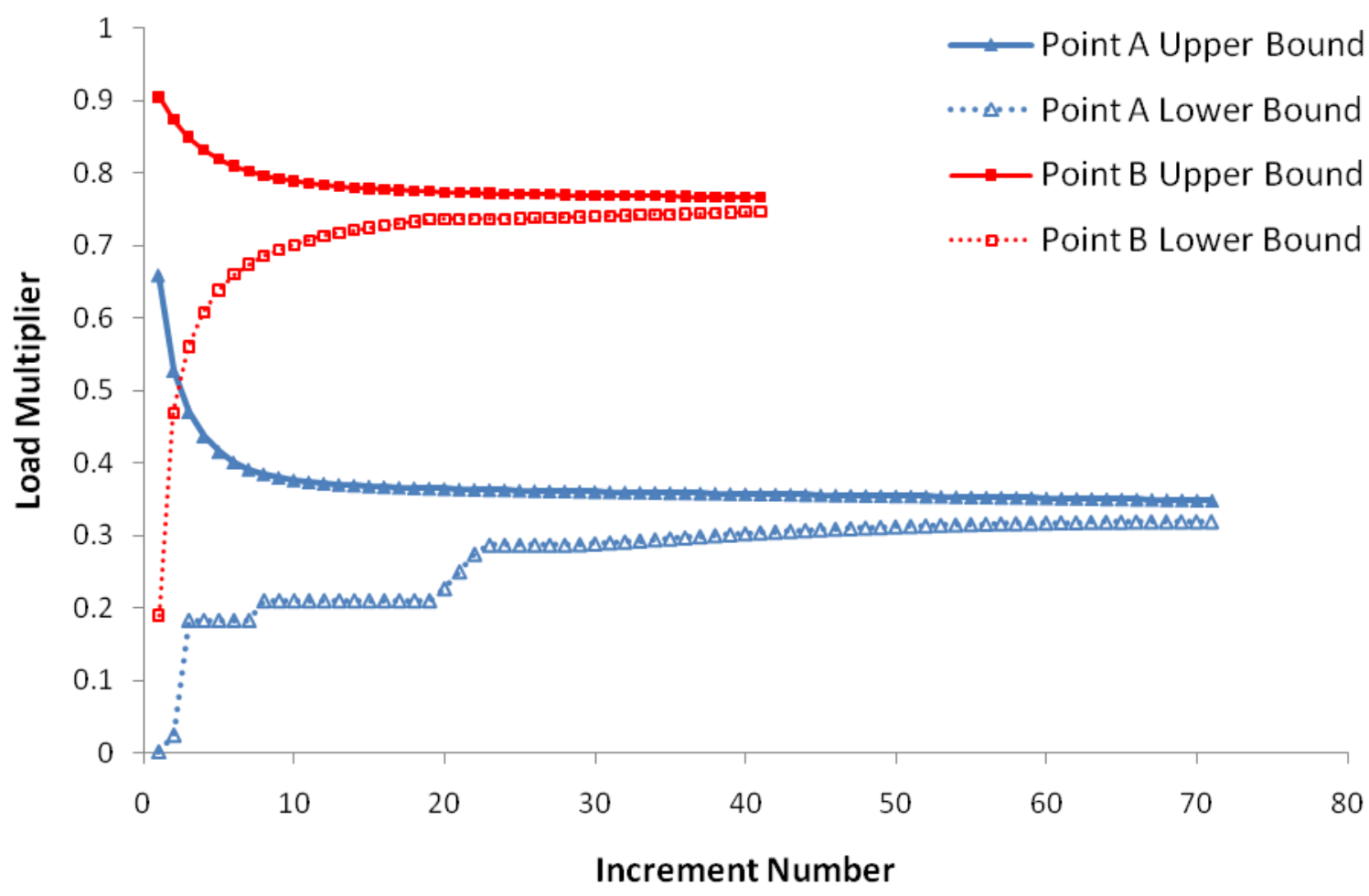

Figure 5 - Convergence of Points $\mathrm{A}$ and $\mathrm{B}$ 